Jadwiga Wolnicka, Institute of Mathematics, Technical University of Łódź, al. Politechniki 11, 93-590, Łódź, Poland.

\title{
QUASI-UNIFORM CONVERGENCE OF SEQUENCES OF 1-IMPROVABLE DISCONTINUOUS FUNCTIONS
}

\begin{abstract}
In the paper it is shown that the strongly quasi-uniform limit of a sequence of 1-improvable discontinuous functions on a complete space $\mathrm{X}$ is a 1-improvable discontinuous function or a continuous function. Automatically the same result will be valid for uniform convergence.
\end{abstract}

Definition 1 ([3]) Let $f: X \rightarrow Y$ (X,Y metric spaces). The function $f$ has at some point $x_{0}$ an improvable discontinuity if $\lim _{x \rightarrow x_{0}} f(x)$ exists and $\lim _{x \rightarrow x_{0}} f(x) \neq f\left(x_{0}\right)$.

In this paper we consider the class $A_{1}$ of real-valued functions $f$ on some metric space $X$ such that the function

$$
f^{(1)}(x)= \begin{cases}\lim _{t \rightarrow x} f(t) & \text { if the limit exists } \\ f(x) & \text { if } \lim _{t \rightarrow x} f(t) \text { doesn't exist }\end{cases}
$$

are continuous.

We shall prove that the strongly quasi-uniform limit of a sequence of functions of the class $A_{1}$ belongs to $A_{1}$. The problem was suggested by $\mathrm{T}$. Świątkowski.

Let $A_{0}$ denote the class of continuous functions. If a function $f \in A_{1} \backslash A_{0}$, then it is called a 1-improvable discontinuous function ([1] and [2]). First we shall consider a subclass $\widetilde{A_{1}}$ of the class $A_{1}$; namely $f \in \widetilde{A_{1}}$ if and only if

$$
\forall_{x \in X} f(x) \geq 0 \text { and } \forall_{x \in X} f^{(1)}(x)=0
$$

Key Words: 1-improvable discontinuous functions, quasi-uniform convergence

Mathematical Reviews subject classification: Primary: 54C30; Secondary 26A15

Received by the editors May 31, 1995 
Lemma 1 Let $f: X \rightarrow \mathbb{R}$ and $f(x)=0$ if $x$ is an isolated point. Then the function $f$ belongs to $\widetilde{A_{1}}$ if and only if

$$
\forall_{x \in X} f(x) \geq 0 \text { and } \forall_{\sigma>0}\left(\{x \in X: f(x) \geq \sigma\}^{d} \cap A(f)=\emptyset\right),
$$

where $A(f)=\{x \in X: f(x)>0\}$. (The notation $\{\cdot\}^{d}$ means the set of limit points of a set).

Proof. First we assume that $f \in \widetilde{A_{1}}$. If $f \in A_{0} \cap \widetilde{A_{1}}$, then $f \equiv 0$ and the above condition is obvious. Assume that $f \in \widetilde{A_{1}} \backslash A_{0}$. Suppose that there exist a real number $\sigma_{0}>0$ and a point $x_{0} \in X$ such that $f\left(x_{0}\right)>0$ and $x_{0} \in\left\{x \in X: f(x) \geq \sigma_{0}\right\}^{d}$. Hence there exists a sequence $\left\{x_{n}\right\}$ such that $\lim _{n \rightarrow \infty} x_{n}=x_{0}$ and $\liminf _{n \rightarrow \infty} f\left(x_{n}\right) \geq \sigma_{0}>0$. Simultaneously $f\left(x_{0}\right)>0$ and $f^{(1)}\left(x_{0}\right)=0$; so $\lim _{x \rightarrow x_{0}} f(x)=0$. Thus we have a contradiction. Hence condition (3) holds.

Now, we assume that condition (3) holds. If $A(f)=\emptyset$, then $f \equiv 0$ and $f \in A_{0} \cap \widetilde{A_{1}}$. Assume that $A(f) \neq \emptyset$. Let us take an arbitrary $x_{0} \in X$. We will consider two cases. First, we assume that $x_{0} \in A(f)$. Then $x_{0} \notin\{x \in X$ : $f(x) \geq \sigma\}^{d}$ for each $\sigma>0$. Thus

$$
\forall_{\sigma>0} \exists_{r>0}\left(K\left(x_{0}, r\right) \backslash\left\{x_{0}\right\}\right) \cap\{x: f(x) \geq \sigma\}=\emptyset,
$$

where $K\left(x_{0}, r\right)=\left\{x \in X: \rho\left(x, x_{0}\right)<r\right\}$. So

$$
\forall_{\sigma>0} \exists_{r>0} \forall_{x \in K\left(x_{0}, r\right) \backslash\left\{x_{0}\right\}}(0 \leq f(x)<\sigma),
$$

which means that $\lim _{x \rightarrow x_{0}} f(x)=0$ and then $f^{(1)}\left(x_{0}\right)=0$.

Now, we assume that $x_{0} \notin A(f)$. If $\lim _{x \rightarrow x_{0}} f(x)$ does not exist, then $f^{(1)}\left(x_{0}\right)=f\left(x_{0}\right)=0$. Assume that $\lim _{x \rightarrow x_{0}} f(x)$ exists and equals $y$ with $y>0$. Then there exists a real number $r>0$ such that $f(x)>y / 2$ for $x \in$ $K\left(x_{0}, r\right) \backslash\left\{x_{0}\right\}$. Let $x^{\prime} \in K\left(x_{0}, r\right) \backslash\left\{x_{0}\right\}$ be arbitrary. There exists a sequence $\left\{x_{n}\right\}$ such that $\lim _{n \rightarrow \infty} x_{n}=x^{\prime}$ and, for each $n \in \mathbb{N}, x_{n} \in K\left(x_{0}, r\right) \backslash\left\{x_{0}\right\}$. Then $x^{\prime} \in\{x: f(x)>y / 2\}^{d} \cap A(f)$, a contradiction. Hence $\lim _{x \rightarrow x_{0}} f(x)=0$ and then $f^{(1)}\left(x_{0}\right)=0$. Thus $f^{(1)}(x)=0$ for each $x \in X$. Hence $f \in \widetilde{A_{1}}$ and the proof is complete.

Definition 2 ([3]) The sequence $\left\{f_{n}\right\}$ is quasi-uniformly convergent on $X$ to $f$ if $f_{n}$ approaches $f$ on $X$ and

$$
\forall_{\epsilon>0} \forall_{n \in \mathbb{N}} \exists_{p \in \mathbb{N}} \forall_{x \in X} \exists_{0 \leq l \leq p}\left|f_{n+l}(x)-f(x)\right|<\epsilon .
$$

Theorem 1 If functions $f_{n}(n=1,2, \ldots)$ belong to $\widetilde{A_{1}}$ and $\left\{f_{n}\right\}$ is quasiuniformly convergent on $X$ to $f$, then $f$ belongs to $\widetilde{A_{1}}$. 
Proof. In view of the above assumption and Lemma 1 we confirm that

$$
\forall_{n \in \mathbb{N}} \forall_{\sigma>0}\left(\left\{x \in X: f_{n}(x) \geq \sigma\right\}^{d} \cap A\left(f_{n}\right)=\emptyset\right),
$$

where $A\left(f_{n}\right)=\left\{x \in X: f_{n}(x)>0\right\}$.

Suppose that $f$ does not belong to $\widetilde{A_{1}}$. Then there exists a real number $\sigma_{0}>0$ such that $\left\{x \in X: f(x) \geq \sigma_{0}\right\}^{d} \cap A(f) \neq \emptyset$. Now let $x_{0} \in A(f)$ and $\left\{x_{k}\right\}$ be a sequence such that $\lim _{k \rightarrow \infty} x_{k}=x_{0}$ and

$$
\forall_{k \in \mathbb{N}}\left(f\left(x_{k}\right) \geq \sigma_{0}\right) .
$$

Put $\epsilon_{0}=1 / 2 \min \left(\sigma_{0}, f\left(x_{0}\right)\right)$. Since the sequence $\left\{f_{n}\left(x_{0}\right)\right\}$ converges to $f\left(x_{0}\right)$, there exists $n^{\prime} \in \mathbb{N}$ such that

$$
\forall_{n>n^{\prime}}\left(f_{n}\left(x_{0}\right)>f\left(x_{0}\right)-\epsilon_{0}\right) .
$$

This follows from the assumption that the sequence $\left\{f_{n}\right\}$ is quasi-uniformly convergent to $f$ on $X$, and thus also on $\left\{x_{k}\right\}$. Therefore there exists a number $p_{0} \in \mathbb{N}$ such that

$$
\forall_{k \in \mathbb{N}} \exists_{0 \leq l \leq p_{0}}\left(f_{n^{\prime}+l}\left(x_{k}\right)>f\left(x_{k}\right)-\epsilon_{0}\right) .
$$

Hence, by (5) and by the selection of $\epsilon_{0}$, we have

$$
\forall_{k \in \mathbb{N}} \exists_{0 \leq l \leq p_{0}}\left(f_{n^{\prime}+l}\left(x_{k}\right)>\sigma_{0} / 2\right) .
$$

Thus there exist $l^{\prime} \in\left\{0,1, \ldots, p_{0}\right\}$ and a subsequence $\left\{x_{k_{m}}\right\}$ of $\left\{x_{k}\right\}$ such that $f_{n^{\prime}+l^{\prime}}\left(x_{k_{m}}\right)>\sigma_{0} / 2$ for each $m \in \mathbb{N}$. Simultaneously, by (6) and by the selection of $\epsilon_{0}, f_{n^{\prime}+l^{\prime}}\left(x_{0}\right)>0$. Then $x_{0} \in\left\{x \in X: f_{n^{\prime}+l^{\prime}}(x) \geq \sigma_{0} / 2\right\}^{d} \cap A\left(f_{n^{\prime}+l^{\prime}}\right)$. This contradicts (4).

Definition 3 ([4]) The sequence $\left\{f_{n}\right\}$ of functions is said to be strongly quasi-uniformly convergent to $f$ on $X$ if every subsequence $\left\{f_{n_{k}}\right\}$ converges quasi-uniformly to $f$.

Theorem I ([5]) For $n \in \mathbb{N}$ let $f_{n}$ be continuous on $X$ and let $\left\{f_{n}\right\}$ converge strongly quasi-uniformly on some dense subset $Z$ of $X$ to a function $f: Z \rightarrow \mathbb{R}$. Then $\left\{f_{n}\right\}$ is strongly quasi-uniformly convergent on $X$ to a function $\varphi$, obviously continuous, whose restriction to $Z$ coincides with $f$.

Theorem 2 For $n \in \mathbb{N}$ let $f_{n}$ belong to the class $A_{1}$ and let $\left\{f_{n}\right\}$ converge strongly quasi-uniformly on a complete space $X$. Then the sequence $\left\{f_{n}^{(1)}\right\}$ (where each $f_{n}^{(1)}$ is defined by formula (1)) is also strongly quasi-uniformly convergent on $X$ to a continuous function $\varphi$. 
Proof. We denote by $E_{n}$ the set of all points in which the function $f_{n}$ has an improvable discontinuity $(n=1,2, \ldots) . E_{n}$ is a set of the first category ([3]). The restriction of $f_{n}$ to $X \backslash E_{n}$ is continuous. Put $E=\bigcup_{n=1}^{\infty} E_{n}$. The set $E$ is of the first category; so $X \backslash E$ is a residual subset of $X$ and since $X$ is complete, $X \backslash E$ is a dense subset of $X$. The sequence $\left\{f_{n}\right\}$ converges strongly quasi-uniformly on $X \backslash E$, and $f_{n}^{(1)}\left|(X \backslash E)=f_{n}\right|(X \backslash E)$. Then the sequence $\left\{f_{n}^{(1)}\right\}$ converges strongly quasi-uniformly on the dense subset $X \backslash E$ of $X$. Since each $f_{n}^{(1)}$ is continuous on $X$, we conclude that $\left\{f_{n}^{(1)}\right\}$ converges strongly quasi-uniformly on $X$ to a continuous function $\varphi$, by Theorem 1 .

Lemma 2 If a function $|f|$ belongs to $\widetilde{A_{1}}$, then $f$ belongs to $A_{1}$ and $f^{(1)}(x)=$ 0 for each $x \in X$.

Proof. Let $E$ denote the set of points in which the function $|f|$ has an improvable discontinuity. Then by assumption, the function $|f|$ has positive values on the set $E$ and is zero on $X \backslash E$. Thus $f(x)=0$ for $x \in X \backslash E$. We shall prove that $f^{(1)}(x)=0$ for each $x \in X$. It suffices to show that $\lim _{t \rightarrow x} f(t)=0$ for each $x \in E$. Obviously, since $x \in E$, we have $\lim _{t \rightarrow x}|f(t)|=0$. So $\lim _{t \rightarrow x} f(t)=0$.

Lemma 3 If a function $f$ belongs to $A_{1}$, then $\left|f-f^{(1)}\right|$ belongs to $\widetilde{A_{1}}$.

We omit the easy proof.

Theorem 3 If for each $n \in \mathbb{N}$ the function $f_{n}$ belongs to $A_{1}$ and $\left\{f_{n}\right\}$ is strongly quasi-uniformly convergent on a complete space $X$ to $f$, then $f$ belongs to $A_{1}$ and $f^{(1)}$ is the strongly quasi-uniform limit of the sequence $\left\{f_{n}^{(1)}\right\}$.

Proof. The sequence $\left\{f_{n}\right\}$ is strongly quasi-uniformly convergent on $X$ to $f$. Then, by Theorem 2, the sequence $\left\{f_{n}^{(1)}\right\}$ is strongly quasi-uniformly convergent on $X$ to a continuous function $\varphi$. Therefore the sequence $\left\{f_{n}-f_{n}^{(1)}\right\}$ is strongly quasi-uniformly convergent on $X$ to $f-\varphi([4])$ and consequently the sequence $\left\{\left|f_{n}-f_{n}^{(1)}\right|\right\}$ is strongly quasi-uniformly convergent on $X$ to $|f-\varphi|$. Note that each function $\left|f_{n}-f_{n}^{(1)}\right|$ belongs to $\widetilde{A_{1}}$ (Lemma 3 ). By Theorem 1 , we have $|f-\varphi| \in \widetilde{A_{1}}$. Then, the function $f-\varphi$ belongs to $A_{1}$ (Lemma 2). Therefore the function $f(f=(f-\varphi)+\varphi)$ belongs to $A_{1}$.

It remains to prove that $f^{(1)}$ is the strongly quasi-uniform limit of the sequence $\left\{f_{n}^{(1)}\right\}$ or $f^{(1)}=\varphi$. Let $f-\varphi=g$. The function $|g| \in \widetilde{A_{1}}$; so by Lemma $2, g^{(1)}(x)=0$ for each $x \in X$. Let $x \in X$ be arbitrary. First, we assume that $\lim _{t \rightarrow x} g(t)$ exists. Then $\lim _{t \rightarrow x} f(t)$ exists and $f^{(1)}(x)=\lim _{t \rightarrow x} f(t)=$ 
$\lim _{t \rightarrow x} g(t)+\lim _{t \rightarrow x} \varphi(t)=0+\varphi(x)=\varphi(x)$. Now, we assume that $\lim _{t \rightarrow x} g(t)$ doesn't exist. Then $\lim _{t \rightarrow x} f(t)$ doesn't exist. Hence $f^{(1)}(x)=f(x)=g(x)+$ $\varphi(x)=0+\varphi(x)=\varphi(x)$. Thus $f^{(1)}(x)=\varphi(x)$ for each $x \in X$.

Definition 4 ([1] $]$ Let $f: X \rightarrow \mathbb{R}$ and let $U(f)=\left\{x \in X: \lim _{t \rightarrow x} f(t) \neq\right.$ $f(x)\}$. For every ordinal $\alpha$ we define a function $f^{(\alpha)}$ by

$$
f^{(\alpha)}(x)= \begin{cases}f(x) & \text { if }\left\{\gamma<\alpha: x \in U\left(f^{(\gamma)}\right)\right\}=\emptyset \\ \lim _{t \rightarrow x} f^{\left(\gamma_{0}\right)}(t) & \text { where } \gamma_{0}=\min \left\{\gamma<\alpha: x \in U\left(f^{(\gamma)}\right)\right\}\end{cases}
$$

$\left(f^{(0)}(x)=f(x)\right.$ for each $\left.x \in X\right)$. We denote by $A_{\alpha}$ the class of functions $f$ such that the function $f^{(\alpha)}$ is continuous. If a function $f \in A_{\alpha} \backslash \bigcup_{0 \leq \beta<\alpha} A_{\beta}$, then it is called an $\alpha$-improvable discontinuous function.

Problem 1 Does Theorem 3 remain valid for sequences of functions of the class $A_{\alpha}$ ?

\section{References}

[1] A. Katafiasz, Improvable functions, Doctoral dissertation, Łódź University, 1993.

[2] A. Katafiasz, Improvable discontinuous functions, Real Analysis Exchange, this issue.

[3] R. Sikorski, Funkcje rzeczywiste, P.W.N. Warszawa, 1958.

[4] T. Świątkowski, On invariants of quasi-uniform convergence, Sc. Bull. of Łódź Technical Univ., Math. 15 (1982).

[5] T. Świạtkowski, Quasi-uniform convergence on dense sets, Sc. Bull. of Śłask Technical Univ., Math.-Phys. 48, Gliwice 1986. 